\title{
Diagnostic utility of abdominal ultrasound in the evaluation of abdomino-pelvic lesions at Bugando Medical Centre in Mwanza, Tanzania
}

\author{
MOHAMED S. BINDE ${ }^{1}$, GODFREY KASANGA ${ }^{2}$, PHILLIPO L. CHALYA ${ }^{1 *}$ and WILLIAM MAHALU ${ }^{3}$ \\ ${ }^{1}$ Department of Surgery, Catholic University of Health and Allied Sciences-Bugando, Mwanza, Tanzania \\ ${ }^{2}$ Department of Radiology, Catholic University of Health and Allied Sciences-Bugando, Mwanza, Tanzania \\ ${ }^{3}$ Department of Cardiothoracic Surgery, Catholic University of Health and Allied Sciences-Bugando, Mwanza, \\ Tanzania
}

\begin{abstract}
Background: Despite the fact that abdominal ultrasound has been widely used in many centres in Tanzania as a primary diagnostic imaging for patients with abdomino-pelvic lesions, its diagnostic utility has not yet been assessed in Tanzania, and therefore its use as an alternative diagnostic tool in patients with abdomino-pelvic lesions is not justified. This study aimed to determine the utility of abdominal ultrasound in the diagnosis of abdomino-pelvic lesions in our local setting.

Methods: This was a cross sectional study to evaluate the diagnostic utility of abdominal ultrasound in patients presenting with abdomino-pelvic lesions at Bugando Medical Centre between January 2017 and July 2017. Abdominal ultrasonographic findings were cross-tabulated against operative findings (the gold standard). Then, the sensitivity, specificity, accuracy, Positive Predictive Value (PPV), Negative Predictive Value (NPV) and accuracy were calculated to determine the diagnostic utility of abdominal ultrasound.

Results: A total of 146 patients ( $M: F$ ratio $=1: 2.2$ ) were studied. The mean age at diagnosis was 39.3 years. The diagnostic utility of abdominal ultrasound in terms of sensitivity, specificity, PPV, NPV and accuracy were $39.4-100 \%, 0-100 \%, 64.7-100 \%, 0-100 \%$ and $40.8-100 \%$ respectively. The kappa statistic ( $k$ ) showed good to excellent agreement with the operative findings, the gold standard $\left(\xi_{=}=0.75-1.00\right)$.

Conclusion: The diagnostic utility of abdominal ultrasound in detecting abdomino-pelvic lesions is much lower than that seen in developed countries, hence, abdominal ultrasound is not a reliable tool in detecting abdomino-pelvic lesions at Bugando Medical Centre and therefore its use should be supplemented with abdominal CT scan in selected cases.
\end{abstract}

Keywords: ultrasound, diagnostic utility, abdomino-pelvic, lesions, Tanzania

\section{Introduction}

Abdomino-pelvic lesions are one of the most common surgical conditions presenting to the surgical wards and clinics in many centres worldwide (Liu et al., 2007). The aetiology of abdomino-pelvic lesions includes a variety of diseases arising from intra-abdominal organs such as the kidneys, liver, gallbladder, pancreas, spleen, aorta and pelvic organs such as the urinary bladder or the ovaries and uterus in women (Liu et al., 2007; Parker et al., 2010). The occurrence of a mass or any lesion in the abdomen creates anxiety for both patients and family members because of fear of probable underlying malignancy (DePriest \& DeSimone, 2003). Therefore, differentiation of benign lesions from malignant abdominal lesions and accurate definitive diagnosis are essential for treatment planning as well as for prognosis for malignant tumours (DePriest \& DeSimone, 2003; McDonald et al., 2010). Understanding the differential diagnosis of abdomino- pelvic lesions plays a major role in defining the appropriate surgical strategy, which in the case of malignancy allows for a longer diseasefree period and even cure (McDonald et al., 2010).

Abdomino-pelvic lesions can present a diagnostic challenge in many resource-poor centres like in Tanzania where late presentation of the disease coupled with lack of sophisticated imaging facilities

\footnotetext{
${ }^{*}$ Correspondence E-mail: plchalya65@gmail.com
} 
required for prompt and precise diagnosis are among the hallmarks of the disease (Aboud et al., 2006). Various advanced imaging modalities such as ultrasound, Computed tomography (CT) scan and magnetic resonance imaging (MRI) are effective for non-invasive evaluation of abdomino-pelvic lesions (Nylund et al., 2017). However, most of these diagnostic imaging are expensive and commonly not available in many centres in resource-limited setting. Following the history and clinical examination, ultrasonography has become one of the first and most useful methods of investigation in many resource-limited centres (Holm et al., 1982; Aboud et al., 2006; McDonald et al., 2010; Nylund et al., 2017). Ultrasonography is an easy to perform, non-invasive, inexpensive and safe imaging technique that is invaluable in Africa where sophisticated diagnostic tools are not readily available. Ultrasonography is a versatile imaging tool, which can evaluate most of the abdomino-pelvic organs; furthermore, it can guide biopsies allowing the cyto-histological and microbiological investigations needed to obtain a definitive diagnosis (Jaeger et al., 1990). Other imaging methods such as Computed Tomography (CT) scan can explore these organs in more detail than ultrasound. However, CT scan may often be considered a second choice in abdominal imaging for the following reasons; it utilizes radiation, it is more expensive, less readily available and often yields results comparable to ultrasound (Chen et al., 2002). This is particularly true in developing countries where the absence or the high cost of the procedure makes abdominal ultrasound a suitable alternative diagnostic tool in the radiological investigation of patients with abdomino-pelvic lesions.

Abdominal ultrasound has been performed in many centres in Tanzania as a primary screening examination for patients with palpable or suspected with abdomino-pelvic lesions (Aboud et al., 2006). However, its diagnostic value has not been evaluated in any hospital in the country including the study area, and therefore its use as an alternative diagnostic tool in patients with abdomino-pelvic lesions is not justified. This existing knowledge gap prompted the authors to conduct this study in our centre to determine the diagnostic value of abdominal ultrasound in patients suspected with abdomino-pelvic lesions. The purpose of this study is to evaluate the clinical utility of abdominal ultrasound in patients suspected with abdomino-pelvic lesions at Bugando Medical Centre, a tertiary care hospital in northwestern Tanzania.

\section{Materials and Methods}

\section{Study design and setting}

This is a cross sectional study to evaluate the diagnostic utility of abdominal ultrasound in patients presenting with abdomino-pelvic lesions at Bugando Medical Centre from January 2017 to July 2017. The study was conducted in the surgical, urological and gynaecological wards and clinics of Bugando Medical Centre. Bugando Medical Centre is the only tertiary health institution in the north-western part of Tanzania, serving a population of about 13 millions. It is an 890 bed referral hospital located in Mwanza city on the southern border of Lake Victoria. It is also a teaching hospital for the Catholic University of Health and Allied Sciences-Bugando. The hospital provides both the outpatient and inpatient surgical and gynaecological services, in addition to medical, paediatric and other health services. The hospital has a department of Radiology where several radiological services including plain x-rays, ultrasound, breast imaging, contrast studies, CT scan are performed. There are no interventional radiological services at the moment probably due to lack of this facility and expertise. There are six operating rooms in the hospital where 20-50 operations, both emergencies and electives are performed daily. The surgical procedures performed include both open and endoscopic surgeries.

\section{Study population}

All patients undergoing abdominal ultrasound for various abdomino-pelvic lesions and subsequently undergo abdomino-pelvic or endoscopic surgery at Bugando Medical Centre during the period of study 
were included in the study. Patients died before surgical treatment and patients who fail to consent for surgical treatment were excluded from the study. Patients without abdominal ultrasound results and those with poor documentation of operative findings were also excluded from the study. The minimum sample size of this study was calculated using Kirkwood \& Sterne (2003) method.

Convenience sampling of patients who met the inclusion criteria was performed until the sample size is reached. Recruitment of patients to participate in the study was done in the surgical, urological and gynaecological wards and clinics of Bugando Medical Centre. All patients presenting with palpable or suspected with abdomino-pelvic masses were screened for inclusion into the study. Patients who met the inclusion criteria were consecutively enrolled in the study after an informed written consent sought from the patients, parents or guardians. All patients included in the study were referred to the Department of Radiology for abdominal ultrasound examination. The ultrasound scans were performed after an overnight fast of at least 12 hours with patients lying supine and using a 3.55.0 MHz frequency curvilinear probe on an ALOKA 1700-SSD ultrasound machine. For examination of the pelvic organs patients were instructed to drink fluids and come for the examination with a full bladder. Prior to scanning, an acoustic gel was applied to the skin of the patient's abdomen to allow the ultrasound probe to glide easily across the skin and to better transmit and receive ultrasonic pulses. The probe was moved around the abdomen's surface to obtain different views of the target areas. Ultrasound scanning was performed by a registered and trained ultrasonographer, either as a technologist or physician (radiologist). All-important sonographic findings were immediately recorded in a special register and a report of the findings were written to the patient's referring doctor. For patients undergoing ultrasound more than once, the most recent result was considered. Depending on the result of ultrasound, all enrolled patients were scheduled for surgery. The surgeries were performed either by a consultant surgeon or a senior resident under the direct supervision of a consultant surgeon. Abdomino-pelvic US findings of all the patients were noted and compared with the actual findings at operation, hence, abdominal US was a tested diagnostic tool whereas surgery was the 'gold standard' test.

\section{Data collection}

Data on each patient were collected and entered into a pretested coded questionnaire prepared for the study. Data entered in the questionnaire included demographic characteristics, clinical and ultrasonographic findings and intra-operative findings of patients.

\section{Data analysis}

The statistical data analysis was performed using statistical package for social sciences (SPSS) version 20 for Windows (SPSS, Chicago IL, USA). The mean (+ standard deviation), median \{+ Interquartile Range) and ranges were calculated for continuous variables whereas proportions and frequency tables were used to summarize categorical variables. Abdominal ultrasonographic findings were crosstabulated against operative findings, the gold standard. Then, the sensitivity, specificity, accuracy, Positive Predictive Value (PPV) and Negative Predictive Value (NPV) and accuracy were calculated to determine the diagnostic utility of abdominal US. The level of significance will be considered as $p<$ 0.05. The Kappa statistic (қ) was calculated to determine the degree of agreement with operative findings. A value above 0.75 suggests excellent agreements with the gold standard (i.e. operative findings), 0.40 to 0.75 is intermediate to good agreement and below 0.40 suggests poor agreement.

\section{Ethical consideration}

The ethical approval to conduct the study was sought from CUHAS/BMC research ethics and review committee. The permission to conduct the study at BMC was sought from hospital authority. Parent or guardian or patient included in the study had to sign a written informed consent before recruitment 
into the study. Confidentiality was assured and the study did not interfere with the decision of the attending surgeon.

\section{Results}

\section{Demographic data}

Between January 2017 and July 2017, a total of 152 patients underwent abdominal ultrasound at Bugando Medical Centre for the diagnosis of abdomino-pelvic lesions. Of these, six patients were excluded from the study due to failure to meet the inclusion criteria. Thus, a total of 146 patients formed the study population. The ages of patients at diagnosis ranged from 6 to 80 years with a mean of $39.3 \pm 17.4$ years. The peak age group was 20-39 years accounting for $61(41.8 \%)$ patients (Figure 1 ). Out of 146 patients, forty five (30.8\%) were males and $101(69.2 \%)$ were females. The male to female ratio was 1:2.2

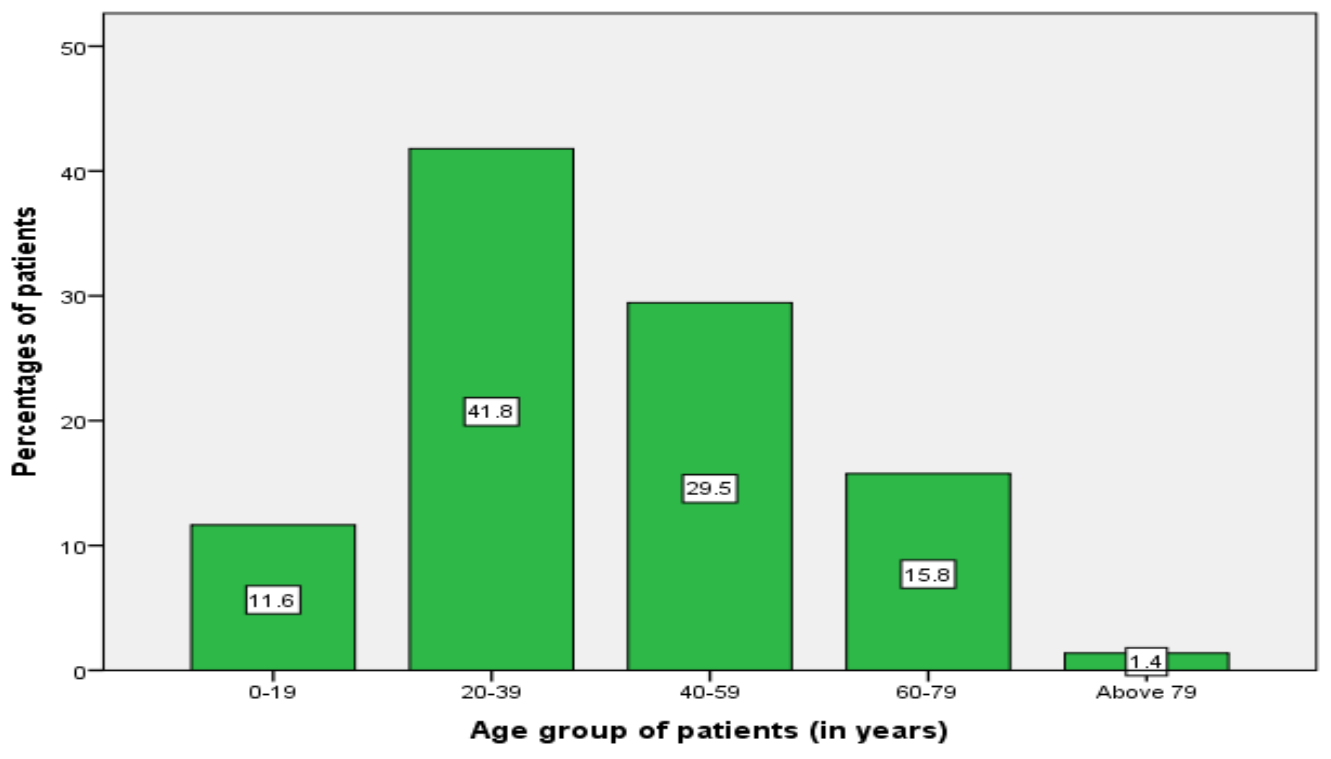

Figure 1: Distribution of patients according to age group

Clinical indications for abdominal ultrasound and diagnoses

Abdominal pain and abdomino-pelvic mass accounted for the most frequent clinical indications for abdominal ultrasound (Table 1).

Table 1: Clinical indications for abdominal ultrasound $(\mathrm{N}=146)$

\begin{tabular}{lll}
\hline Clinical indications for abdominal ultrasound & Frequency & Percentage \\
\hline Abdominal pain & 47 & 32.2 \\
Abdomino-pelvic mass & 44 & 30.1 \\
Lower urinary tract symptoms & 12 & 8.2 \\
Abdominal pain and abdomino-pelvic mass & 5 & 3.4 \\
Abdominal pain and vagina bleeding & 4 & 2.7 \\
Other symptoms & 34 & 23.3 \\
Total & 146 & 100.0 \\
\hline
\end{tabular}


Out of the 146 patients suspected with abdomino-pelvic lesions, gynaecological lesions were the most common lesions accounting for $30.1 \%$ of cases, followed by gastrointestinal and urinary tract lesion (Table 2).

Table 2: Distribution of patients according to the clinical diagnosis $(\mathrm{N}=146)$

\begin{tabular}{lll}
\hline Abdomino-pelvic lesions & Frequency & Percentage \\
\hline Gastrointestinal lesions & 38 & 26.0 \\
Intra-abdominal mass & 12 & \\
Peritonitis /perforated viscera & 8 & \\
Splenic tumour & 5 & \\
Appendicitis/appendicular abscess & 4 & \\
Bowel obstruction & 3 & \\
Intussusceptions & 3 & 21.2 \\
Pyloric stenosis & 3 & \\
Urinary tract lesions & 31 & \\
Obstructive uropathy & 15 & \\
Urinary bladder tumours & 7 & \\
Renal tumour & 7 & \\
Cystitis & 1 & \\
Urinary bladder stones & 1 & \\
Hepato-biliary lesions & 12 & \\
Obstructive jaundice & 6 & 30.1 \\
Gall bladder tumour & 4 & \\
Cholecystitis & 2 & \\
Gynaecological lesions & 44 & \\
Ectopic pregnancy & 12 & \\
Pelvic abscess & 11 & \\
Uterine fibroid & 10 & \\
Tubo-ovarian mass & 5 & \\
Endometriosis /endometrial hyperplasia & 3 & \\
Chronic pelvic pain & 2 & \\
Pelvic inflammatory disease & 1 & \\
Abdominal trauma lesions & 23 & \\
Visceral injuries & 23 & \\
Other abdomino-pelvic lesions & 10 & \\
\hline
\end{tabular}

\section{Abdominal sonographic findings}

Abdominal ultrasound done in 146 patients revealed abnormal finding in 116 (79.5\%) patients. Table 3 below shows distribution of patients according to abdominal sonographic findings

Table 3: Distribution of patients according to abdominal sonographic findings ( $N=146)$

\begin{tabular}{cll}
\hline Abdominal sonographic findings & Frequency & Percentage \\
\hline Gastro-intestinal lesions & 31 & 21.2 \\
- Splenic tumour & 5 & \\
- Bowel obstruction & 5 & \\
- Intra-abdominal mass & 4 & \\
- Intussusceptions & 4 & \\
- Peritonitis & 4 & \\
- Pastric tumour & 3 & \\
- Umbic stenosis & 3 & \\
\hline
\end{tabular}




\begin{tabular}{cll}
\hline Urinary tract lesions & 23 & 15.8 \\
- BPH & 11 & \\
- Urinary bladder tumour & 9 & \\
- Urinary bladder stone & 3 & 5.5 \\
Hepato-biliary lesions & 8 & \\
- Cholelithiasis & 3 & \\
- Choll bladder tumour & 2 & \\
- Acalculus cholecystitis & 2 & 25.3 \\
Gynaecological lesions & 1 & \\
- Uterine fibroid & 37 & \\
- Ectopic pregnancy & 8 & \\
- Tubo-ovarian mass & 7 & \\
- Ovarian cyst & 6 & \\
- Pelvic abscess & 5 & \\
Abdominal traumatic lesions & 4 & \\
- Splenic injury & 2 & \\
- Urinary bladder injury & 17 & \\
- Renal injury & 13 & \\
Normal scan & 2 & \\
\hline
\end{tabular}

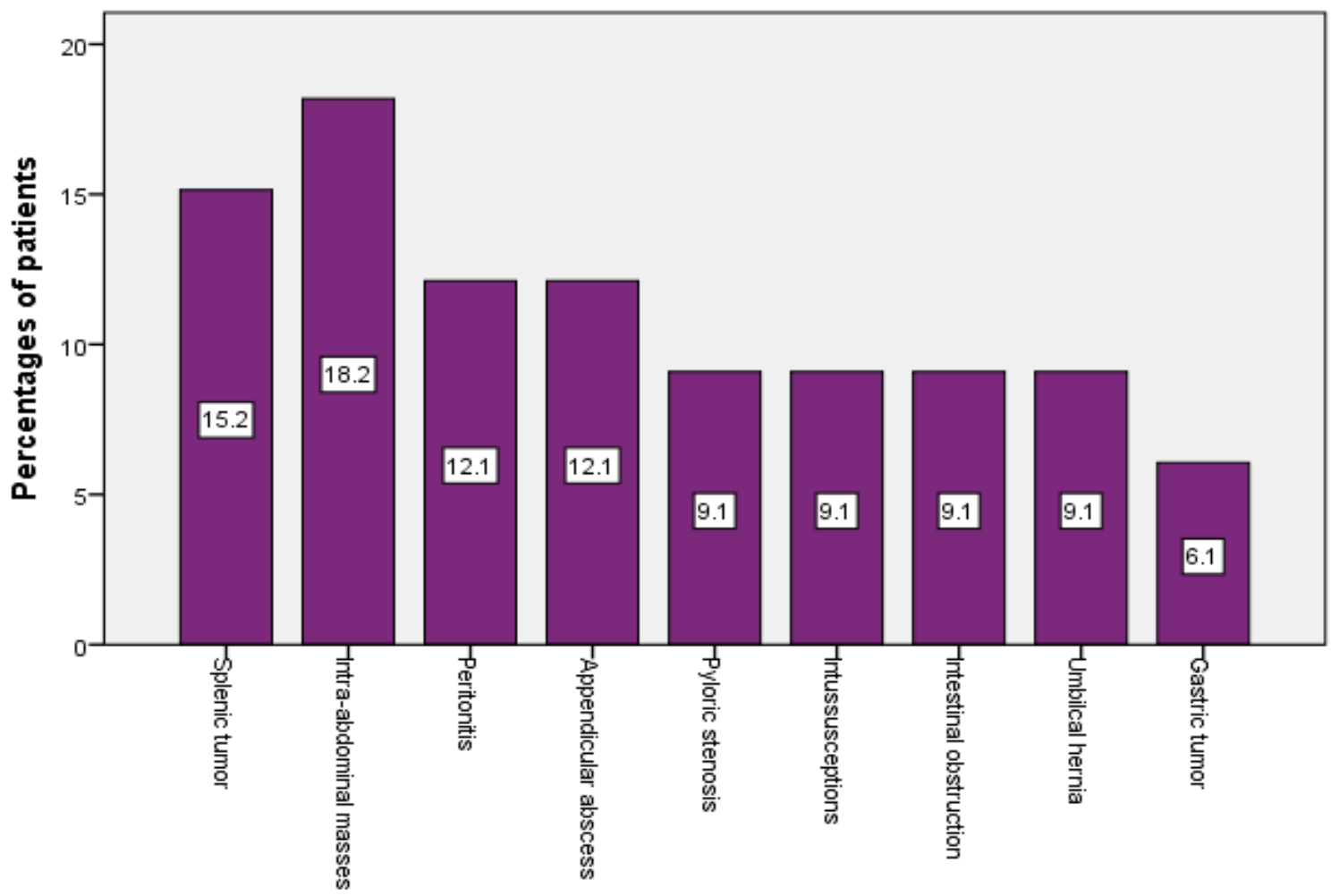

Figure 2: Distribution of patients according to operative findings (gastro-intestinal lesions) 


\section{Operative findings}

All the 146 patients underwent surgical procedures for their abdomino-pelvic lesions. The majority of them, 99(67.8\%) were operated electively, while 47(32.2\%) patients had an emergency surgery that required immediate resuscitation. Intra-abdominal tumour (Figure 2) followed by splenic tumour accounted for the majority of the gastro-intestinal lesion when analysed by operative findings.

Table 4: Distribution of patients according to operative/cystoscopic findings (hepato-biliary, urinary tract and abdominal traumatic lesions)

\begin{tabular}{lll}
\hline Operative findings & Frequency & Percentage \\
\hline Hepato-biliary lesions & 6 & 4.1 \\
- Gall bladder stones & 5 & \\
- Gall bladder tumour & 1 & 21.2 \\
Urinary tract lesions & 31 & \\
- Urinary bladder tumour & 12 & \\
- BPH & 10 & \\
- Cystitis & 4 & \\
- Urinary bladder stone & 3 & \\
- Ureteric stenosis & 1 & \\
- Hydronephrosis & 1 & \\
Abdominal traumatic lesions & 11 & \\
- Hemoperitoneum & 5 & \\
- Splenic injury & 4 & $\mathbf{1 7 . 8}$ \\
- Renal injury & 1 & \\
- Ureteric injury & 1 & \\
No lesions found & $\mathbf{2 6}$ & \\
\hline
\end{tabular}

Urinary tract lesions (21.2\%) accounted for the majority of patients in the category of hepato-biliary, urinary tract and abdominal traumatic lesions (Table 4). On the other hand, uterine fibroid (21.6\%) and ectopic pregnancy (13.5\%) formed the largest proportion in the group of gynaecological lesions (Figure 3).
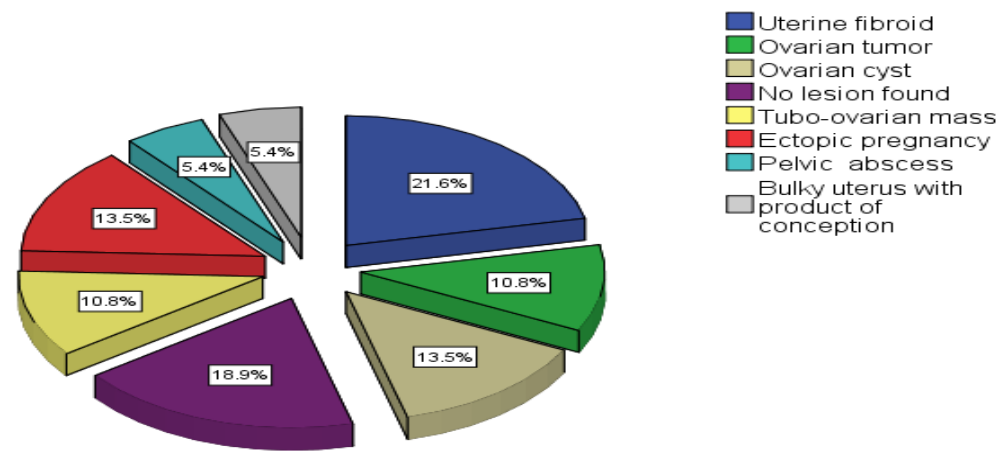

Figure 3: Distribution of patients according to operative findings (Gynaecological lesions)

Diagnostic value of abdominal ultrasound in specific abdomino-pelvic diseases

The sensitivity, specificity, Positive predictive value (PPV), Negative predictive value (NPV) and accuracy of abdominal ultrasound in detecting gastrointestinal tract, hepatobiliary tract, urinary tract, 
gynaecological and abdominal trauma lesions were (71.6-100\%, 0-83.0\%, 72.6-80.4\%, 68.9-79.4\% and 70.8-82.8\%), (87.9-90.2\%, 62.4-67.5\%, 76.0-80.0\%, 71.9-72.8 and 76.8-100\%), (100\%, 64.2-100\%, 85.0-100\%, $82.4-100 \%$ and $78.9-100 \%),(68.8-89.1 \%, 76.1-82.2 \%, 76.6-84.8 \%, 62.6-100 \%$ and $70.4-83.5 \%)$ and (38.4\%, $50.6 \%, 64.7 \%, 30.2 \%$ and $40.8 \%$ ) respectively.

\section{Discussion}

In this study, abdomino-pelvic diseases were found to be most common in the third decade of life and tended to affect more females than males. Similar demographic observations have also been reported by other authors (Brunetti et al., 2006; Aboud et al., 2006; Liu et al., 2007). Female predominance in the present study is due to the large number of gynaecological patients presenting with abdominopelvic diseases. The clinical indications for abdominal ultrasound in our patients is not different from those in other studies (Tshibwabwa et al., 2000; Aboud et al., 2006; Brunetti et al., 2006), with abdominal pain being common to all the patients followed by abdomino-pelvic masses. Also lower urinary tract symptoms were the most common indications in urological patients.

In keeping with other studies done elsewhere (Tshibwabwa et al., 2000; Brunetti et al., 2006), abdominal ultrasound in our study revealed abnormal findings in more than three quarter of patients the majority being gynaecological patients. In the present study, gynaecological diseases mainly uterine fibroids, ectopic pregnancy and tubo-ovarian masses were also the most common operative findings. Abdominal ultrasound plays a major role of investing gynaecological lesions such as uterine fibroids, endometrial cancer, tubo-ovarian masses, pelvic inflammatory diseases and ectopic pregnancy (Grab et al., 2000; Valentin, 2004). In this study, the diagnostic accuracy of abdominal ultrasound in detecting uterine fibroid was high, comparable to findings by other studies (Valentin, 2004; Geomini et al., 2006). This high diagnostic accuracy of abdominal ultrasound can be attributed to the fact that the majority of our patients in this region present late with huge fibroids and therefore the diagnosis of uterine fibroid was straightforward. The diagnostic value of abdominal ultrasound may further be improved by the use of trans-vaginal ultrasound.

In the present study, ectopic pregnancy accounted for $17.6 \%$ of cases. The diagnostic utility of abdominal sound in detecting ectopic concurs with findings from other studies (Grab et al., 2000; Valentin, 2004; Kirk, 2012; Casikar et al., 2012). The diagnostic accuracy of abdominal ultrasound in detecting ectopic pregnancy may also be improved by the use of trans-vaginal ultrasound, and may even further by the use of colour-flow Doppler ultrasound. Ultrasound is also the primary imaging tool for a patient considered to have ovarian masses. However, ultrasound images may not be helpful for differentiating hydrosalpinx, paraovarian and tubal cysts from ovarian (Timor-Tritsch et al., 1998). The diagnostic accuracy of abdominal ultrasound in detecting ovarian masses was found to be similar with other studies (Grab et al., 2000; Valentin, 2004).

Abdominal ultrasound has been reported to have a sensitivity of more than $80 \%$ and a specificity of $95 \%$ in the diagnosis of acute appendicitis without perforation (Alfraih et al., 2014). In this study, the diagnostic utility of abdominal ultrasound in diagnosis of acute appendicitis was quite comparable to the findings of the other studies (Pinto et al., 2013; Alfraih et al., 2014). Abdominal ultrasound has been advocated that it should be used routinely in an attempt to reduce the negative appendectomy rate, to patient with equivocal clinical findings and young women, in whom it will exclude a gynaecological cause for pain. In addition, abdominal ultrasound has a role in the management of intestinal obstruction. In this study it was found to have high diagnostic accuracy, sensitivity and PPV. This observation was comparable with other studies (Scheible \& Goldberger, 1979; Nicolaou et al., 2005). Ultrasound is a valuable tool in the diagnosis of intestinal obstruction, as it can differentiate between mechanical and functional intestinal obstruction. 
Urinary bladder tumour was the commonest urinary tract disease accounting for $38.7 \%$ of all urological diseases in this study. Tumours of the urinary bladder are better detected by transabdominal ultrasound. Trans-abdominal ultrasound has a sensitivity of 61 to $72 \%$, in detecting tumours of the urinary bladder. The combination of the trans-abdominal and trans-rectal ultrasound increases the sensitivity to $95 \%$ (Feeney \& Anderson, 2011). In our study, the sensitivity, specificity, PPV, NPV and accuracy of abdominal ultrasound in detecting urinary bladder tumours were $100 \%$ each, respectively.

In this study, the diagnostic accuracy of abdominal ultrasound in detecting splenic injury was low. Similar observations have been reported by other authors (Bode et al., 1993; McElveen \& Collin, 1997; Ma et al., 2001; Brown et al., 2003; Nural et al., 2005). This diagnostic value is too low to make ultrasound a reliable investigation tool in evaluating patients with ruptured spleen at BMC. Successful use of abdominal ultrasound in the setting of trauma can be maximized with adequate sonographer training, appreciation of technical limitations, and adherence to an appropriate trauma ultrasound protocol (Bode et al., 1993; McElveen \& Collin, 1997; Ma et al., 2001). Focused assessment for the sonographic examination of the trauma patient (FAST) is currently advocated in the management of abdominal trauma to improve the diagnostic accuracy of abdominal ultrasound in the setting of trauma (Brown et al., 2003; Nural et al., 2005). FAST accurately detects the presence of hemoperitoneum in patients with blunt trauma, it does not readily identify intraparenchymal or retroperitoneal injuries. Therefore, a computed tomographic scan of the abdomen may be needed to complement the FAST and reduce the incidence of missed injuries (Makama et al., 2012).

The major limitations of this study is due to the fact that abdominal ultrasound is an operator dependent and may be obscured by air, so inter-observer variation in reporting the findings may have affected our results. Also, lack of FAST in our centre may have affected the diagnostic utility of abdominal ultrasound in trauma patients. Small sample size was also a major limitation in this study.

In conclusion, this study demonstrated that the diagnostic utility of abdominal ultrasound in detecting abdomino-pelvic lesions is much lower than that reported in high-income countries, hence, abdominal ultrasound is not a reliable tool in detecting abdomino-pelvic lesions at Bugando Medical Centre and therefore should be supplemented with abdominal CT scan in selected cases. FAST should be established in the management of abdominal trauma at Bugando Medical Centre in order to improve the diagnostic accuracy of abdominal ultrasound in our setting.

\section{Acknowledgements}

We would like to express our sincerest gratitude and thanks to all those who participated in the preparation of this manuscript and those who were involved in the care of our study patients

\section{References}

Aboud, M.M., Mkony, C. \& Wuster, M.M.D. (2006) Elective Abdominal Ultrasonography by Surgeons at MNH, Dar-es-Salaam, Tanzania. East and Central African Journal of Surgery 11: 52-56.

Alfraih, Y., Postuma, R. \& Keijzer, R. (2014) How do you diagnose appendicitis? An international evaluation of methods. International Journal of Surgery 12: 67-70.

Bode, P.J., Niezen, R.A., van Vugt, A.B. \& Schipper, J. (1993) Abdominal ultrasound as a reliable indicator for conclusive laparotomy in blunt abdominal trauma. Journal of Trauma 34: 27-31.

Brown, M.A., Sirlin, C.B., Hoyt, D.B. \& Casola, G. (2003) Screening ultrasound in blunt abdominal trauma. Journal of Intensive Care Medicine 18: 253-260.

Brunetti, E., Brigada, R., Poletti, F., Maiocchi, L., Garlaschelli, A., Gulizia, R. \& Filice, C. (2006) The current role of abdominal ultrasound in the clinical management of patients with AIDS. Ultraschall in der Medizin 27: 20-33. 
Casikar, I., Reid, S. \& Condous, G. (2012) Ectopic pregnancy: Ultrasound diagnosis in modern management. Clinical Obstetrics and Gynecology 55: 402-409.

Chen, S.C., Yen, Z.S., Wang, H.P., Lin, F.Y., Hsu, C.Y., Chen, W.J. (2002) Ultrasonography is superior to plain radiography in the diagnosis of pneumoperitoneum. British Journal of Surgery 89, 351354.

DePriest, P.D. \& DeSimone, C.P. (2003) Ultrasound screening for the early detection of ovarian cancer. Journal of Clinical Oncology 21: 194-199.

Feeney, D.A. \& Anderson, K.L. (2011) Radiographic imaging in urinary tract disease. In: Bartges J, Polzin DJ, eds. Nephrology and Urology of Small Animals. Ames, IA: Wiley-Blackwell; 97-127.

Geomini, P.M., Kluivers, K.B., Moret, E., Bremer, G.L., Kruitwagen, R.F. \& Mo, B.W. (2006) Evaluation of adnexal masses with three-dimensional ultrasonography. Obstetrics and Gynaecology 108: 1167-1175.

Grab, D., Flock, F., Stohr, I., Nussle, K., Rieber, A., Fenchel., S., Brambs, H.J., Reske, S.N. \& Kreienberg R. (2000) Classification of asymptomatic adnexal masses by ultrasound, magnetic resonance imaging, and positron emission tomography. Gynaecological Oncology 77: 454-459.

Holm, H.H., Gammelgaard, J., Jensen, F., Smith, E.H. \& Hillman, B.J. (1982) Ultrasound in the diagnosis of palpable abdominal mass. A prospective study of 107 patients. Gastrointestinal Radiology 2: 149-151.

Jaeger, H.J., Macfie, J., Mitchell, C.J., Couse, N. \& Wai, D. (1990) Diagnosis of abdominal masses with percutaneous biopsy guided by ultrasound. BMJ 301: 1335 .

Kirkwood, B.R. \& Sterne, J.A. (2003) editors. Calculation of required sample size. Essential medical statistics. 2nd ed. Oxford: Blackwell Science; 413-428.

Kirk, E. (2012) Ultrasound in the diagnosis of ectopic pregnancy. Clinical Obstetrics and Gynecology 55: 395-401.

Liu, J., Xu, Y. \& Wang, J. (2007) Ultrasonography, computed tomography and magnetic resonance imaging for diagnosis of ovarian carcinoma. European Journal of Radiology 62: 328-334.

Ma, O.J., Kefer, M.P., Stevison, K.F. \& Mateer, J.R. (2001) Operative versus non-operative management of blunt abdominal trauma: Role of ultrasound-measured intraperitoneal fluid levels. American Journal of Emergency Medicine 19: 284-286.

McDonald, J.M., Doran, S., DeSimone, C.P., Ueland, F.R., DePriest, P.D., Ware, R.A., Saunders, B.A., Pavlik, E.J., Goodrich, S., Kryscio, R.J. \& van Nagell, J.R., Jr.(2010) Predicting risk of malignancy in adnexal masses. Obstetrics and Gynecology 115: 687-694.

McElveen, T.S. \& Collin, G.R. (1997) The role of ultrasonography in blunt abdominal trauma: A prospective study. American Journal of Surgery 63: 184-8.

Nicolaou, S., Kai, B., Ho, S., Su, J. \& Ahamed, K. (2005) Imaging of acute small-bowel obstruction. AJR American Journal of Roentgenology 185: 1036-1044.Nural, M.S., Yardan, T., Guven, H., Baydin, A., Bayrak, I.K. \& Kati, C. (2005) Diagnostic value of ultrasonography in the evaluation of blunt abdominal trauma. Diagnostic Interventional Radiology 11: 41-44.

Nylund, K., Maconi, G., Hollerweger, A., Ripolles, T., Pallotta, N., Higginson, A., Serra C., Dietrich, C.F., Sporea, I., Saftoiu, A., Dirks, K., Hausken, T., Calabrese, E., Romanini, L., Maaser, C., Nuernberg, D. \& Gilja, O.H. (2017) EFSUMB Recommendations and Guidelines for Gastrointestinal Ultrasound. Ultraschall in der Medizin 38: e1-e15

Parker, L., Levin, D.C., Frangos, A. \& Rao, V.M. (2010) Geographic variation in the utilization of noninvasive diagnostic imaging: national Medicare data, 1998-2007. American Journal of Roentgenology 194: 1034-1039.

Pinto, F., Pinto, A., Russo, A., Coppolino, F., Bracale, R., Fonio, P., Macarini, L. \& Giganti, M. (2013) Accuracy of ultrasonography in the diagnosis of acute appendicitis in adult patients: review of the literature. Critical Ultrasound Journal 5: S1-S2. 
Scheible, W. \& Goldberger, L.E. (1979) Diagnosis of small bowel obstruction: The contribution of diagnostic ultrasound. AJR American Journal of Roentgenology 133: 685-688.

Timor-Tritsch, I.E., Lerner, J.P., Monteagudo, A., Murphy, K.E. \& Heller, D.S. (1998) Transvaginal sonographic markers of tubal inflammatory disease. Ultrasound in Obstetrics and Gynecology 12: 56-66.

Tshibwabwa, E.T., Mwaba, P., Bogle-Taylor, J. \& Zumla, A. (2000) Four-year study of abdominal ultrasound in 900 Central African adults with AIDS referred for diagnostic imaging. Abdominal Imaging 25: 290-296.

Valentin, L. (2004) Use of morphology to characterize and manage common adnexal masses. Best Practical Research in Clinical Obstetrics and Gynaecology 18: 71 